\title{
A new era for junior doctor roster management for the medical directorate in Musgrove Park Hospital, Taunton: Ensuring equity, contractual compliance and pro-active safe staffing across all clinical areas. A work in progress.
}

\author{
Author: Rachael Ward ${ }^{*}$
}

\section{Defining the problem}

There are two types of rosters for junior doctors working in the medical directorate: one for their in-hours commitments; one for their out-of-hours commitments. The out-of-hours roster is set from a rota template coordinated by central admin staff. In-hours rosters are needed for each separate clinical area to ensure safe staffing levels. They need to account for: out-of-hours commitments; annual leave; study leave; rest days.

Musgrove Park Hospital (MPH) in-hours rosters are created and selfpoliced by junior doctors. Audit demonstrated:

> departments fail to ensure $100 \%$ minimum staffing in each clinical area

> not all juniors are getting the correct annual leave/study leave/ zero days

$>$ there is no robust system for demonstrating contractual compliance

> there is no clear structure of clinical responsibility for the in- and out-of-hours medical rosters

> the lack of communication between the two rosters means that processes for anticipating gaps and filling emergency gaps are inefficient.

\section{Aims}

> Achieve $95 \%$ (of $100 \%$ standard) of minimum staffing across all medical wards by August 2019.

> Ensure in-hours rosters are contractually compliant by April 2019.

$>$ Ensure contractual compliance is visible and easily audited.

> Agree and implement a standard structure of responsibility for in- and out-of-hours rosters by August 2019.

\section{Methods}

Quality improvement methodology was used. Survey and semistructured interviews were conducted with relevant staff to help

Author: Taunton and Somerset NHS Foundation Trust; Musgrove Park Hospital

${ }^{*} \mathrm{RCP}$ chief registrar to characterise the problem. Process maps were created to gain greater understanding.

Audit was undertaken in the largest department (Care of the Elderly) to quantify the problem for the pilot.

A rota tool was developed and piloted with the following functionality.

> Each department's roster is built on a single spreadsheet using a uniform template (customised for each department), visible on the intranet.

> For each day the number of juniors on the ward automatically tallies to give a visual display of staffing levels: red, below minimum; amber, at minimum; green, above minimum.

> The ward staffing levels automatically tally to a central dashboard giving a complete oversight of staffing in the directorate.

> Each junior's individual leave, study leave and rest days automatically tally and go red if they exceed their allowance.

$>$ Cross cover between departments is visually displayed.

$>$ Prospective roster building and sharing.

Additionally, policies have been drafted documenting rota coordinator responsibilities and cross cover policies. A contract explanation document aids rota coordinators.

\section{Early results}

Initial audit showed that the directorate could only prove that $33 \%$ of juniors had taken the correct annual leave, $27 \%$ had taken the correct rest days. This has improved to $100 \%$. A survey has shown that $100 \%$ of participants think that the system has had a positive impact on patient safety and staff wellbeing, reduced days below minimum staffing and increased confidence in filling rota gaps.

\section{Scope}

The project is immensely exciting. Improved visibility of staffing provides data for workforce planning not previously available. The trust is currently scoping how best to support medical staff with the maintenance and building of the rosters. 